\title{
Ubiquitin ligase E6-AP and its role in human disease
}

\author{
Konstantin Matentzoglu* and Martin Scheffner* ${ }^{\mathbf{1}}$ \\ "Laboratory of Cellular Biochemistry, Department of Biology, University of Konstanz, 78457 Konstanz, Germany, and †Konstanz Research School Chemical \\ Biology, University of Konstanz, 78457 Konstanz, Germany
}

\begin{abstract}
The ubiquitin ligase E6-AP (E6-associated protein) represents a prime example for the notion that deregulated modification of proteins with ubiquitin contributes to the development of human disease: loss of E6-AP function by mutation is responsible for the development of AS (Angelman syndrome), a neurological disorder, and unscheduled activation of E6-AP by complex formation with the E6 oncoprotein of HPVs (human papillomaviruses) contributes to cervical carcinogenesis. However, while there is a considerable amount of data concerning the oncogenic properties of the E6-E6-AP complex, only little is known about the function(s) of E6-AP in neurons. This is mainly due to the fact that although some E6-AP substrates have been identified, it is at present unclear whether deregulated modification/degradation of these proteins is involved in the pathogenesis of AS. Similarly, the cellular pathways involving E6-AP remain enigmatic. To obtain insights into the physiological functions of E6-AP, we are currently employing several strategies, including quantitative affinity proteomics and RNA interference approaches. The results obtained will eventually allow the introduction of E6-AP into functional protein networks and so reveal potential targets for molecular approaches in the treatment of E6-AP-associated diseases.
\end{abstract}

\section{Introduction}

The ubiquitin ligase E6-AP (E6-associated protein) represents the founding member of the HECT (homologous with E6-associated protein C-terminus) family of E3 ubiquitin ligases [1-3]. E6-AP is encoded by the $U B E 3 A$ gene located on chromosome 15q11-13 [4-7]. The UBE3A gene encodes three protein isoforms generated by differential splicing [8]. These isoforms differ at their very $\mathrm{N}$-termini and consist of 852 amino acids (isoform 1), 872 amino acids (isoform 3) and 875 amino acids (isoform 2 ) respectively (in the following, numbering of amino acid residues is according to isoform 1). It is at present unknown whether the isoforms have different properties (e.g. towards putative target proteins) and/or show tissue-specific expression. Intriguingly, deregulation of the E3 activity of E6-AP has been associated with two distinct human diseases $([6,7]$, reviewed in $[9,10])$ and, thus, E6-AP may currently represent the most prominent example for the notion that inappropriate modulation of the activity of components of the ubiquitin-conjugation system contributes to the development of human disease. Unscheduled activation of E6-AP contributes to cervical carcinogenesis ('gain of function'), whereas inactivation results in a neurodevelopmental disorder, the AS (Angelman syndrome; 'loss of function')

Key words: Angelman syndrome, E6-associated protein (E6-AP), E6 oncoprotein, human papillomavirus (HPV), neurodevelopmental disorder, ubiquitin ligase. Abbreviations used: AS, Angelman syndrome; E6-AP, E6-associated protein; HECT, homologous with E6-associated protein C-terminus; HPV, human papillomavirus; PWS, Prader-Willi syndrome; RNAi, RNA interference.

${ }^{1}$ To whom any correspondence should be addressed (email martin.scheffner@unikonstanz.de).
(Figure 1B). Taken together, these facts necessitate the thorough elucidation of cellular pathways involving E6-AP.

\section{'Gain of function': E6-AP and cervical cancer}

It is generally accepted that infection with certain types of HPVs (human papillomaviruses) represents the most significant risk factor for the development of cervical cancer (for a review, see [9]). Approx. $30 \mathrm{HPV}$ types have been associated with lesions of the anogenital tract and these HPVs can be roughly classified into 'low risk' and 'high risk' types based on their association with clinical lesions. Whereas low-risk HPVs are generally associated with benign lesions including condyloma accuminata, high-risk HPVs have been aetiologically associated with malignant lesions of the anogenital tract, most notably cervical cancer. High-risk HPVs encode two major oncoproteins termed E6 and E7, and the respective genes are the only viral genes that are generally retained and expressed in cervical cancer tissues. Furthermore, a number of studies have shown that continuous expression of both E6 and E7 is required for the viability of HPV-positive cancer cells (for reviews, see [9-11]). Since cervical cancer represents the second most common cancer in women, it has been, and still is, of considerable interest to elucidate and characterize the oncogenic functions of E6 and E7.

E6-AP was originally isolated as a protein that binds to the E6 oncoprotein of HPVs associated with cervical cancer and, in complex with E6, targets the tumour suppressor p53 for ubiquitination and proteasome-mediated degradation $[1,12]$. The region comprising 378-401 amino acids of 
Figure 1| E6-AP and human disease

(A) Schematic illustration of the domain structure of E6-AP isoform 1. The E6-binding site and the HECT domain are depicted (the asterisk indicates the active cysteine residue at position 820 of the HECT domain). (B) E6AP in human disease. Inappropriate activation of E6-AP contributes to cervical carcinogenesis ('gain of function'), while inactivation results in a neurodevelopmental disease, the AS ('loss of function').

A

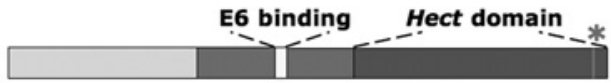

B

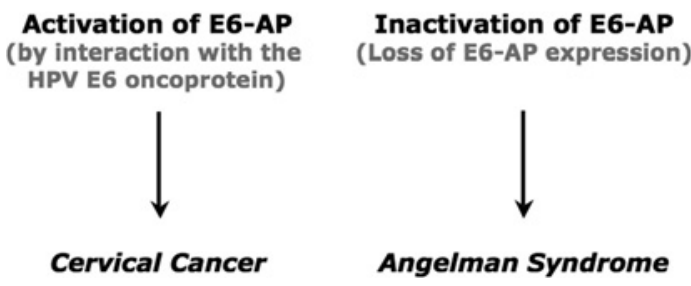

E6-AP serves as a binding site for HPV E6 proteins [13] (Figure 1A). This region is necessary and sufficient for the E6-E6-AP interaction and even subtle changes within this region interfere with E6 binding [14]. However, p53 is not the only target of the E6-E6-AP complex. Besides p53, known substrates of the E6-E6-AP complex include several PDZ-domain-containing proteins (e.g. hDlg and Scribble), E6TP1 and NFX1-91, a transcriptional repressor of the gene encoding hTERT, the rate-limiting catalytic subunit of telomerase [15-20] (for reviews, see [10,11]).

The notion that the ability of E6 to utilize E6-AP to target p53 and other cellular proteins for degradation contributes to its oncogenic functions is supported by several observations: (i) transgenic mouse models indicate that both ablation of p53 activity and the interaction with PDZ domain proteins are critical for HPV-induced tumorigenesis [21,22]; (ii) in contrast with many other tumour types, the p53 gene is very rarely mutated in cervical carcinomas $[9,10]$. Thus E6-E6-AP-induced degradation of p53 can be considered as functionally equivalent to inactivation of $\mathrm{p} 53$ by mutation of the p53 gene, although the situation in HPV-positive cancers may be somewhat more complicated (for a detailed discussion of this issue, see [10]); and (iii) interference with E6-AP expression by antisense RNA-based approaches or by RNAi (RNA interference) results in accumulation of p53 and activation of its transcriptional and growth-suppressive properties in HPV-positive but not in HPV-negative cells (indicating that E6-AP plays no or only a minor role in p53 degradation in the absence of E6) [23-26].

Finally, it should be noted that E6-AP may not only be utilized by E6 but, in addition, may represent a direct target for E6, since binding of E6 targets E6-AP for ubiquitination and degradation [27]. Although the physiological relevance of this finding is not yet understood, it can be speculated that an E6-induced decrease in intracellular E6-AP levels should have profound effects on the stability of E6-independent substrates of E6-AP.

\section{'Loss of function': E6-AP and AS}

AS was first described in 1965 by the paediatrician Harry Angelman [28,29]. AS is a genetic disorder with an incidence in the range of approx. 1 in 10000 to 1 in 40000 and is characterized by mental retardation, movement or balance disorder, characteristic abnormal behaviours and severe limitations in speech and language. Development of AS has been linked to chromosome 15q11-13, which is known as the PWS (Prader-Willi syndrome)/AS region and, as indicated above, contains the UBE3A gene. The PWS/AS region comprises approx. 4 megabases and contains a bipartite imprinting centre and, thus, maternally and paternally imprinted genes [30]. The PWS and the AS represent two clinically distinct neurodevelopmental disorders with PWS resulting from paternal genetic deficiency and AS from maternal genetic deficiency [5]. Importantly, the paternal UBE3A allele is imprinted in certain brain areas (Purkinje neurons and a subset of hippocampal neurons), but expressed from both alleles in all other tissues throughout the body [31,32]. Furthermore, all of the genetic abnormalities associated with AS affect the expression of the maternal UBE3A gene and/or the E3 activity of E6-AP. Genetic abnormalities include deletion of the 15q11-13 region of the maternal chromosome (accounting for approx. $70 \%$ of the AS cases), uniparental paternal disomy, defects in imprinting and single-point mutations in the UBE3A gene [5,29,33]. However, why loss of E6AP activity results in AS development is not understood, mainly because the cellular pathways and processes that are controlled by E6-AP have not yet been identified. In this context, it should be noted that cell culture studies indicate that E6-AP positively affects the activity of nuclear hormone receptors (oestrogen, androgen and progesterone receptors) and that this property of E6-AP is not related to its E3 function [34]. The effect on hormone receptors appears to be mediated via two spatially separated amino acid motifs (one within the E6-binding site and the other within the HECT domain) of E6-AP that match the consensus sequence of a nuclear hormone receptor interaction motif (LXXLL) [34,35]. However, the relevance of the ability of E6-AP to affect nuclear hormone receptor activity for the development of AS is unclear (e.g. this property is not affected in those E6-AP mutants that are derived from AS patients with point mutations in the UBE3A gene).

Finally, deregulation of E6-AP expression/activity has also been shown to contribute to autism (e.g. [36,37]). However, the available data are purely descriptive and, apart from altered expression profiles, do not provide any evidence for a direct contribution of deregulated E6-AP activity to the development of autism.

\section{E6-AP in mice}

Two Ube3a-knockout mouse models (Ube3a ${ }^{\text {tm1 Alb }}$ and Ube $\left.3 \mathrm{a}^{\mathrm{tm} 1 \mathrm{Jwf}}\right)$ are currently available $[38,39]$. The respective 
mice display a number of features reminiscent of AS patients including motor dysfunction, mental retardation, seizures and sleep disturbances [38-40]. Remarkably, Ube3a transgenic mice develop these AS-like features by disruption of only the maternal allele, thus reflecting the situation in AS patients. Indeed, similar to the human UBE3A gene, the mouse Ube3a gene is biallelically expressed in all somatic cells with the exception of Purkinje cells (cerebellum), hippocampal neurons and mitral cells of the olfactory bulb, in which the paternally derived Ube3a gene is silenced [41].

The abovementioned data indicate that Ube3a-knockout mice provide an invaluable tool for the elucidation of the molecular mechanisms underlying the neurodevelopmental defects of AS patients. This notion is underlined by experiments studying ataxia, one of the diagnostic criteria of AS. In general, ataxia can result from various dysfunctions of the cerebellar cortex. In Ube3a-knockout mice, the cortical dysfunction may arise from fast oscillation (approx. $160 \mathrm{~Hz}$ ) in the cerebellar cortex, which is sustained by an abnormally increased firing rate and rhythmicity of (E6-AP-deficient) Purkinje cells [42]. This oscillation is inhibited by sensory stimulation or by gap junction and $\mathrm{GABA}_{\mathrm{A}}(\gamma$-aminobutyric acid type A) receptor blockers respectively. Of note, a similar oscillation was previously found in mice lacking calciumbinding proteins that also present ataxia, but never in wildtype mice [43]. Thus it has been proposed that fast oscillation in the cerebellar cortex is implicated in the cerebellar symptomatology of AS and, thus, inhibition of this oscillation might alleviate symptoms of ataxia in AS patients [42].

\section{'E6-APomics'}

To understand why loss of the E3 activity of E6-AP results in the development of AS, it is essential to identify the cellular pathways that involve E6-AP. An obvious strategy to do so is the identification and characterization of proteins that interact with E6-AP and modulate its function and/or serve as ubiquitination substrates of E6-AP. Several E6-independent substrates of E6-AP have been reported, including HHR23A and HHR23B (the human homologues of Saccharomyces cerevisiae RAD23), Blk (a member of the Src family of non-receptor tyrosine kinases), Bak (human pro-apoptotic protein), $\mathrm{Mcm} 7$ (which is involved in DNA replication) and AIB1 (co-activator of steroid receptors) [44-48]. However, it is at present unclear whether deregulated degradation of these proteins is involved in the pathogenesis of AS patients. In addition, in one of the Ube3a-knockout mouse models, cytoplasmic levels of p53 have been reported to be significantly increased in post-mitotic neurons [38]. However, since this was not observed in the other Ube3a-knockout mouse model [39] and since all available data obtained in cell culture systems indicate that, in the absence of HPV E6, E6-AP does not play a prominent role in $\mathrm{p} 53$ degradation, it seems likely that the observed increase in $\mathrm{p} 53$ levels is an indirect rather than a direct effect of loss of E6-AP expression.

To identify cellular pathways involving E6-AP, we are currently using a combination of approaches, including mouse models, RNAi-based strategies and quantitative proteomics
Figure 2 | Schematic representation of the experimental workflow used to elucidate the cellular functions of E6-AP

For details, see the text. MEF, mouse embryonic fibroblast.

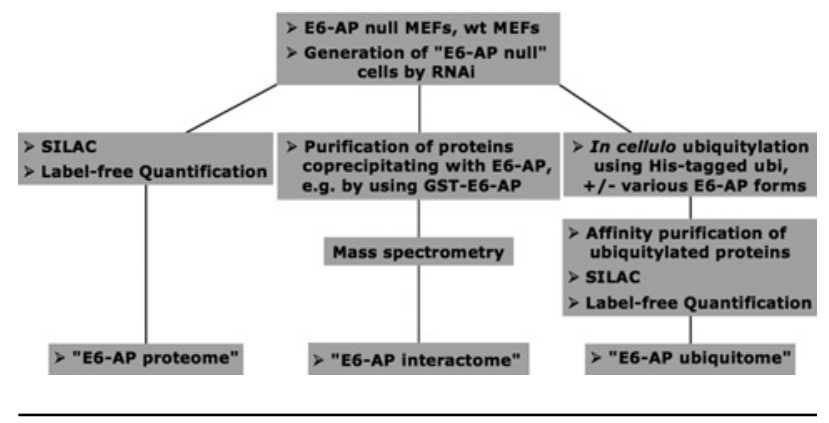

(Figure 2). We have developed an analysis pipeline along three major workflows. First, we have performed classical affinity proteomics (with recombinantly expressed E6-AP as matrix) using protein extracts derived from either Ube3a-knockout mice or from human cell lines, in which endogenous E6-AP expression is constitutively or inducibly ablated by RNAi. By this approach, we have been able to identify several putative interaction partners of E6-AP, where some appear to directly bind to E6-AP and others indirectly. Secondly, we are using comparative MS-based proteome analysis using both SILAC (stable isotope labelling in cell culture) [49] and label-free quantification [50]. For this, we are employing both MEFs (mouse embryonic fibroblasts) derived from either wild-type or Ube3a-knockout mice and matched human cell lines that either express E6-AP or do not express E6-AP (see above). So far, we have been able to identify approx. 100 proteins that appear to be altered in their expression levels as a consequence of loss of E6-AP expression. Thirdly, we are employing quantitative MS approaches to identify potential ubiquitination substrates of E6-AP by performing in cellulo ubiquitination assays combined with affinity purification. In addition, ectopic expression of E6-AP is highly cytotoxic to cells. Thus we have generated inducible overexpression systems for E6AP that will be used in RNAi screens to identify proteins that are involved in mediating the cytotoxic effect of E6AP. Finally, when the datasets for the different approaches are available, we will attempt to establish a semantic bioinformatics approach to cross-compare the individual datasets. This will eventually enable us to place E6-AP into a network of pathways that are relevant for the physiological and pathophysiological functions of E6-AP.

\section{E6-AP: a therapeutic target?}

The data available suggest that the ability of the HPV E6 oncoprotein to target p53 and other cellular proteins for degradation is required for the viability of cell lines derived from cervical cancer patients [26,51]. Thus the E6-E6-AP complex represents a bona fide target for molecular approaches in the treatment of cervical cancer. However, since E6-AP is expressed in all human tissues, E6 (since it is a viral protein) rather than E6-AP should be the target of choice for potential anticancer strategies. 
AS is caused by loss of E6-AP expression, indicating that proteins acting upstream or downstream of pathways involving E6-AP rather than E6-AP represent targets for potential therapeutic approaches. However, AS is a neurodevelopmental disorder and it is at present unclear whether the neuronal defects caused by loss of E6-AP activity can be reverted and, if so, at which stage of neuronal development. As a first step to answer this question, the availability of Ube3Aknockout mice should prove helpful. For example, it has been reported that hippocampal slices derived from Ube3Aknockout mice show deficits in long-term potentiation [38]. If it is possible to rescue this phenotype by restoration of E6AP expression, this would suggest that it should be possible to devise strategies to improve the conditions of AS patients.

Work in our laboratory has been supported by the Deutsche Forschungsgemeinschaft, the EU (European Union) Network of Excellence RUBICON and the German-Israeli Foundation for Scientific Research and Development.

\section{References}

1 Scheffner, M., Huibregtse, J.M., Vierstra, R.D. and Howley, P.M. (1993) The HPV-16 E6 and E6-AP complex functions as a ubiquitin-protein ligase in the ubiquitylation of $\mathrm{p} 53$. Cell 75, 495-505

2 Huibregtse, J.M., Scheffner, M., Beaudenon, S. and Howley, P.M. (1995) A family of proteins structurally and functionally related to the E6-AP ubiquitin-protein ligase. Proc. Natl. Acad. Sci. U.S.A. 92, 2563-2567

3 Scheffner, M., Nuber, U. and Huibregtse, J.M. (1995) Protein ubiquitination involving an E1-E2-E3 enzyme ubiquitin thioester cascade. Nature 373, 81-83

4 Nakao, M., Sutcliffe, J.S., Durtschi, B., Mutirangura, A., Ledbetter, D.H. and Beaudet, A.L. (1994) Imprinting analysis of three genes in the Prader-Willi/Angelman region: SNRPN, E6-associated protein, and PAR-2 (D15S225E). Hum. Mol. Genet. 3, 309-315

5 Nicholls, R.D. and Knepper, J.L. (2001) Genome organization, function, and imprinting in Prader-Willi and Angelman syndromes. Annu. Rev. Genomics Hum. Genet. 2, 153-175

6 Kishino, T., Lalande, M. and Wagstaff, J. (1997) UBE3A/E6-AP mutations cause Angelman syndrome. Nat. Genet. 15, 70-73

7 Matsuura, T., Sutcliffe, J.S., Fang, P., Galjaard, R.J., Jiang, Y.H., Benton, C.S., Rommens, J.M. and Beaudet, A.L. (1997) De novo truncating mutations in E6-AP ubiquitin-protein ligase gene (UBE3A) in Angelman syndrome. Nat. Genet. 15, 74-77

8 Yamamoto, Y., Huibregtse, J.M. and Howley, P.M. (1997) The human E6-AP gene (UBE3A) encodes three potential protein isoforms generated by differential splicing. Genomics 41, 263-266

9 zur Hausen, H. (2002) Papillomaviruses and cancer: from basic studies to clinical application. Nat. Rev. Cancer 2, 342-350

10 Scheffner, M. and Whitaker, N.). (2003) Human papillomavirus induced carcinogenesis and the ubiquitin-proteasome system. Semin. Cancer Biol. 13, 59-67

11 Mantovani, F. and Banks, L. (2001) The human papillomavirus E6 protein and its contribution to malignant progression. Oncogene 20,7874-7887

12 Huibregtse, J.M., Scheffner, M. and Howley, P.M. (1993) Cloning and expression of the cDNA for E6-AP, a protein that mediates the interaction of the human papillomavirus E6 oncoprotein with p53. Mol. Cell. Biol. 13, 775-784

13 Huibregtse, J.M., Scheffner, M. and Howley, P.M. (1993) Localization of the E6-AP regions that direct human papillomavirus E6 binding, association with p53, and ubiquitination of associated proteins. Mol. Cell. Biol. 13, 4918-4927

14 Be, X., Hong, Y., Wei, J., Androphy, E.J., Chen, J.J. and Baleja, J.D. (2001) Solution structure determination and mutational analysis of the papillomavirus E6 interacting peptide of E6AP. Biochemistry $\mathbf{4 0}$ 1293-1299
15 Nakagawa, S. and Huibregtse, J.M. (2000) Human scribble (Vartul) is targeted for ubiquitin-mediated degradation by the high-risk papillomavirus E6 proteins and the E6AP ubiquitin-protein ligase. Mol. Cell. Biol. 20, 8244-8253

16 Gewin, L., Myers, H., Kiyono, T. and Galloway, D.A. (2004) Identification of a novel telomerase repressor that interacts with the human papillomavirus type-16 E6/E6-AP complex. Genes Dev. 18, 2269-2282

17 Töpffer, S., Müller-Schiffmann, A., Matentzoglu, K., Scheffner, M. and Steger, G. (2007) Protein tyrosine phosphatase $\mathrm{H} 1$ is a target of the E6 oncoprotein of high-risk genital human papillomaviruses. J. Gen. Virol. 88, 2956-2965

18 Kuballa, P., Matentzoglu, K. and Scheffner, M. (2007) The role of the ubiquitin ligase E6-AP in human papillomavirus E6-mediated degradation of PDZ domain-containing proteins. J. Biol. Chem. 5, 65-71

19 Jing, M., Bohl, J., Brimer, N., Kinter, M. and Vande Pol, S.B. (2007) Degradation of tyrosine phosphatase PTPN3 (PTPH1) by association with oncogenic human papillomavirus E6 proteins. J. Virol. 81, 2231-2239

20 Gao, Q., Kumar, A., Singh, L., Huibregtse, J.M., Beaudenon, S., Srinivasan, S., Wazer, D.E., Band, H. and Band, V. (2002) Human papillomavirus E6-induced degradation of E6TP1 is mediated by E6AP ubiquitin ligase. Cancer Res. 62, 3315-3321

21 Pan, H. and Griep, A.E. (1994) Altered cell cycle regulation in the lens of HPV-16 E6 or E7 transgenic mice: implications for tumor suppressor gene function in development. Genes Dev. 8, 1285-1299

22 Simonson, S.J., Difilippantonio, M.J. and Lambert, P.F. (2005) Two distinct activities contribute to human papillomavirus 16 E6's oncogenic potential. Cancer Res. 65, 8266-8273

23 Beer-Romero, P., Glass, S. and Rolfe, M. (1997) Antisense targeting of E6AP elevates p53 in HPV-infected cells but not in normal cells. Oncogene 14, 595-602

24 Kim, Y., Cairns, M.J., Marouga, R. and Sun, L.Q. (2003) E6AP gene suppression and characterization with in vitro selected hammerhead ribozymes. Cancer Gene Ther. 10, 707-716

25 Kelley, M.L., Keiger, K.E., Lee, C.J. and Huibregtse, J.M. (2005) The global transcriptional effects of the human papillomavirus E6 protein in cervica carcinoma cell lines are mediated by the E6AP ubiquitin ligase. J. Virol. 79, 3737-3747

26 Hengstermann, A., D'silva, M.A., Kuballa, P., Butz, K., Hoppe-Seyler, F. and Scheffner, M. (2005) Growth suppression induced by downregulation of E6-AP expression in human papillomavirus-positive cancer cell lines depends on p53. J. Virol. 79 , 9296-9300

27 Kao, W.H., Beaudenon, S.L., Talis, A.L., Huibregtse, J.M. and Howley, P.M. (2000) Human papillomavirus type 16 E6 induces self-ubiquitination of the E6AP ubiquitin-protein ligase. J. Virol. 74, 6408-6417

28 Angelman, H. (1965) 'Puppet children': a report of three cases. Dev. Med. Child Neurol. 7, 681-688

29 Clayton-Smith, J. and Laan, L. (2003) Angelman syndrome: a review of the clinical and genetic aspects. J. Med. Genet. 40, 87-95

30 Amos-Landgraf, J.M., Ji, Y., Gottlieb, W., Depinet, T., Wandstrat, A.E., Cassidy, S.B., Driscoll, D.J., Rogan, P.K., Schwartz, S. and Nicholls, R.D. (1999) Chromosome breakage in the Prader-Willi and Angelman syndromes involves recombination between large, transcribed repeats at proximal and distal breakpoints. Am. J. Hum. Genet. $\mathbf{6 5}$, 370-386

$31 \mathrm{Vu}$, T.H. and Hoffman, A.R. (1997) Imprinting of the Angelman syndrome gene, UBE3A, is restricted to brain. Nat. Genet. 17, 12-13

32 Rougeulle, C., Glatt, H. and Lalande, M. (1997) The Angelman syndrome candidate gene, UBE3A/E6-AP, is imprinted in brain. Nat. Genet. 17, 14-15

33 Fang, P. Lev-Lehman, E., Tsai, T.F., Matsuura, T., Benton, C.S., Sutcliffe, J.S., Christian, S.L., Kubota, T., Halley, D.J. and Meijers-Heijboer, H. et al (1999) The spectrum of mutations in UBE3A causing Angelman syndrome. Hum. Mol. Genet. 8, 129-135

34 Nawaz, Z., Lonard, D.M., Smith, C.L., Lev-Lehman, E., Tsai, S.Y., Tsai, M.J. and O'Malley, B.W. (1999) The Angelman syndrome-associated protein, E6-AP, is a coactivator for the nuclear hormone receptor superfamily. Mol. Cell. Biol. 19, 1182-1189

35 Smith, C.L., DeVera, D.G., Lamb, D.J., Nawaz, Z., liang, Y.H., Beaudet, A.L. and O'Malley, B.W. (2002) Genetic ablation of the steroid receptor coactivator-ubiquitin ligase, E6-AP, results in tissue-selective steroid hormone resistance and defects in reproduction. Mol. Cell. Biol. 22 525-535

36 Jordan, C. and Francke, U. (2006) Ube3a expression is not altered in Mecp2 mutant mice. Hum. Mol. Genet. 15, 2210-2215 
37 Samaco, R.C., Hogart, A. and LaSalle, J.M. (2005) Epigenetic overlap in autism-spectrum neurodevelopmental disorders: MECP2 deficiency causes reduced expression of UBE3A and GABRB3. Hum. Mol. Genet. 14 483-492

38 Jiang, Y.H., Armstrong, D., Albrecht, U., Atkins, C.M., Noebels, J.L., Eichele, G., Sweatt, J.D. and Beaudet, A.L. (1998) Mutation of the Angelman ubiquitin ligase in mice causes increased cytoplasmic p53 and deficits of contextual learning and long-term potentiation. Neuron 21, 799-811

39 Miura, K., Kishino, T., Li, E., Webber, H., Dikkes, P., Holmes, G.L. and Wagstaff, J. (2002) Neurobehavioral and electroencephalographic abnormalities in Ube3a maternal-deficient mice. Neurobiol. Dis. $\mathbf{9}$ 149-159

40 Colas, D., Wagstaff, J., Fort, P., Salvert, D. and Sarda, N. (2005) Sleep disturbances in Ube3a maternal-deficient mice modeling Angelman syndrome. Neurobiol. Dis. 20, 471-478

41 Albrecht, U., Sutcliffe, J.S., Cattanach, B.M., Beechey, C.V., Armstrong, D., Eichele, G. and Beaudet, A.L. (1997) Imprinted expression of the murine Angelman syndrome gene, Ube3a, in hippocampal and Purkinje neurons. Nat. Genet. 17, 75-78

42 Cheron, G., Servais, L., Wagstaff, J. and Dan, B. (2005) Fast cerebellar oscillation associated with ataxia in a mouse model of Angelman syndrome. Neuroscience 130, 631-637

43 Cheron, G., Servais, L., Dan, B., Gall, D., Roussel, C. and Schiffmann, S.N. (2005) Fast oscillation in the cerebellar cortex of calcium binding protein-deficient mice: a new sensorimotor arrest rhythm. Prog. Brain Res. 148, 165-180

44 Kumar, S., Talis, A.L. and Howley, P.M. (1999) Identification of HHR23A as a substrate for $E 6$-associated protein-mediated ubiquitination. J. Biol. Chem. 274, 18785-18792
45 Oda, H., Kumar, S. and Howley, P.M. (1999) Regulation of the Sic family tyrosine kinase Blk through E6AP-mediated ubiquitination. Proc. Natl. Acad. Sci. U.S.A. 96, 9557-9562

46 Thomas, M. and Banks, L. (1998) Inhibition of Bak-induced apoptosis by HPV-18 E6. Oncogene 17, 2943-2954

47 Kuhne, C. and Banks, L. (1998) E3-ubiquitin ligase/E6-AP links multicopy maintenance protein 7 to the ubiquitination pathway by a novel motif, the L2G box. J. Biol. Chem. 273, 34302-34309

48 Mani, A., Oh, A.S., Bowden, E.T., Lahusen, T., Lorick, K.L., Weissman, A.M., Schlegel, R., Wellstein, A. and Riegel, A.T. (2006) E6AP mediates regulated proteasomal degradation of the nuclear receptor coactivator Amplified in Breast Cancer 1 in immortalized cells. Cancer Res. 66, 8680-8686

49 Ong, S.E., Blagoev, B., Kratchmarova, I., Kristensen, D.B., Steen, H., Pandey, A. and Mann, M. (2002) Stable isotope labeling by amino acids in cell culture, SILAC, as a simple and accurate approach to expression proteomics. Mol. Cell. Proteomics 1, 376-386

50 Silva, J.C., Denny, R., Dorschel, C.A., Gorenstein, M., Kass, I.J., Li, G.Z., McKenna, T., Nold, M.J., Richardson, K., Young, P. and Geromanos, S. (2005) Quantitative proteomic analysis by accurate mass retention time pairs. Anal. Chem. 77, 2187-2200

51 Butz, K., Denk, C., Ullmann, A., Scheffner, M. and Hoppe-Seyler, F. (2000) Induction of apoptosis in human papillomavirus-positive cancer cells by peptide aptamers targeting the viral E6 oncoprotein. Proc. Natl. Acad. Sci. U.S.A. 97, 6693-6697 